\title{
Recaída de Variabilidade Operante com a Combinação dos Procedimentos de Renovação, Restabelecimento e Ressurgência*
}

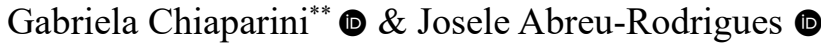 \\ Universidade de Brasília, Brasília, DF, Brasil
}

\begin{abstract}
RESUMO - O reaparecimento de variabilidade comportamental previamente extinta (i.e., recaída) foi investigado por meio dos modelos experimentais de renovação, restabelecimento e ressurgência. Na Fase de Treino (contexto A), ao serem expostos ao esquema múltiplo Lag 10 Acoplado, ratos apresentaram níveis similares de variabilidade nos dois componentes. Na Fase de Eliminação (contexto B), o esquema múltiplo Repetição Repetição promoveu a extinção da variabilidade. $\mathrm{Na}$ Fase de Teste (contexto A), com a suspensão da contingência de repetição e a liberação de reforços independentes, a variabilidade reapareceu no componente "Lag 10", anteriormente correlacionado com reforçamento da variação, mas não no componente "Acoplado". Esse resultado sugere que a variabilidade observada no teste de recaída corresponde à variabilidade operante, e não à variabilidade induzida pela extinção.
\end{abstract}

PALAVRAS-CHAVE: variabilidade, recaída, renovação, restabelecimento, ressurgência

\section{Relapse of Operant Variability with the Combination of the Renewal, Reinstatement, and Resurgence Procedures}

\begin{abstract}
The reappearance of previously extinguished behavioral variability (i.e. relapse) was investigated with three experimental models: renewal, reinstatement and resurgence. In the Training Phase (context A), when exposed to the multiple Lag 10 Yoke schedule, rats showed similar levels of variability in both components. In the Elimination Phase (context B), the multiple Repetition schedule promoted the extinction of variability. In the Test Phase (context A), with the suspension of the repetition contingency and the delivery of response-independent reinforcers, variability reappeared in the "Lag 10" component, the one previously correlated with the reinforcement of variation, but not in the "Yoke" component. This result suggests that the variability observed in the relapse test corresponded to operant variability, and not to extinction-induced variability.
\end{abstract}

KEYWORDS: variability, relapse, renewal, reinstatement, resurgence

Comportamentos reforçados, e posteriormente extintos, podem reaparecer diante de certas condições ambientais. Esse fenômeno é denominado de recaída comportamental (Epstein, 1983) e tem sido investigado por meio de modelos experimentais que se distinguem em termos das variáveis de controle. No modelo de renovação, uma resposta (alvo) é reforçada no contexto A (Fase de Treino) e extinta no contexto B (Fase de Eliminação); na Fase de Teste, a extinção permanece em vigor, mas há uma alteração de contexto (e.g., retorno ao contexto A, mudança para o contexto C). Nesta última fase, a resposta alvo tende a reaparecer em decorrência da mudança para um contexto não previamente correlacionado com extinção (e.g., Berry et al., 2014; Podlesnik et al., 2017; Skinner, 1950; Todd et al., 2012).

O modelo experimental do restabelecimento, assim como o de renovação, compreende três fases. Na Fase de Treino, ocorre o reforçamento da resposta alvo, e na Fase de Eliminação, essa resposta é extinta. Na Fase de Teste, a resposta alvo continua sem produzir reforços, mas reforços independentes do responder são liberados. A liberação desses reforços é comumente acompanhada pelo reaparecimento da resposta alvo, sugerindo que a recaída é ocasionada pelas propriedades discriminativas do reforço (e.g., Doughty

\footnotetext{
* Esse artigo é derivado da dissertação de mestrado da primeira autora.

** E-mail: gabi.chiaparini@gmail.com

n Submetido: 04/11/2019; Revisado: 05/06/2020; Aceito: 04/08/2020.
} 
et al., 2004; Miranda-Dukoski et al., 2015; Reid, 1958; Souza \& Abreu-Rodrigues, 2010). Finalmente, no modelo de ressurgência, a resposta alvo também é inicialmente reforçada (Fase de Treino) e, em seguida, extinta (Fase de Eliminação). Diferentemente dos modelos anteriores, no entanto, uma resposta alternativa é reforçada nessa segunda fase. Na Fase de Teste, ambas as respostas não produzem reforços. Observa-se a recaída da resposta alvo, a qual é atribuída à descontinuação dos reforços para a resposta alternativa (e.g., Craig et al., 2017; Silva et al., 2008; Epstein, 1983; Nighbor et al., 2020).

Alguns estudos têm utilizado combinações dos modelos de recaída. Bouton e Trask (2016), por exemplo, combinaram os modelos de restabelecimento e ressurgência, com ratos. Na Fase de Treino, a resposta de pressão à barra direita (ou esquerda) foi reforçada com comida de acordo com o esquema de intervalo variável (VI) $30 \mathrm{~s}$. Na Fase de Eliminação, a resposta alvo estava em extinção e uma resposta alternativa (pressionar uma barra diferente daquela utilizada na Fase de Treino) foi reforçada com sucralose de acordo com o esquema VI $30 \mathrm{~s}$. Na Fase de Teste, as respostas alvo e alternativa estavam em extinção. Além disso, reforços independentes de qualquer resposta foram liberados de acordo com o esquema de tempo randômico (RT) 30 s: para o Grupo Reforço 1, o reforço era comida, e para o Grupo Reforço 2, era sucralose. Para o Grupo Nenhum, não houve liberação de reforços independentes. O reaparecimento da resposta alvo ocorreu quando os reforços correlacionados com o treino da resposta alvo foram reintroduzidos (Grupo 1), ou quando não havia reforços (Grupo Nenhum), mas não quando reforços correlacionados com a extinção da resposta alvo foram liberados (Grupo 2).

A literatura mostra recaída não somente de unidades comportamentais compostas por uma única resposta (e.g., pressionar uma barra), mas também por sequências de respostas (e.g. emitir uma sequência de respostas distribuídas em duas barras). A recaída de sequências de respostas foi observada com sequências contendo duas (Bachá-Mendez et al., 2007), três (Sánchez-Carrasco \& Nieto, 2005; Pontes $\&$ Abreu-Rodrigues, 2016), quatro (Galizio et al., 2018) e cinco (Pontes \& Abreu-Rodrigues, 2016) respostas. Os efeitos do número de respostas por sequência (custo do responder) sobre a ressurgência foi investigado por Pontes e Abreu-Rodrigues, com estudantes universitários. Na Fase de Treino, uma sequência alvo contendo cinco (Experimento 1) ou três (Experimento 2) respostas foi reforçada de acordo com o esquema CRF. Na Fase de Eliminação, a sequência alvo foi colocada em extinção e uma sequência alternativa foi continuamente reforçada. Na Fase de Teste, nenhuma sequência era reforçada. Houve maior reaparecimento da sequência alvo que compreendia três respostas (menor custo) do que daquela com cinco respostas (maior custo).

O presente estudo também utilizou sequências de respostas como unidade comportamental, mas o foco da investigação consistiu na recaída da variabilidade na emissão de sequências de respostas. Galizio et al.
(2018) publicaram o primeiro estudo sobre o tema. No Experimento 2, os autores avaliaram o restabelecimento de variabilidade comportamental, com pombos. Na Fase de Treino, sequências de quatro respostas foram reforçadas de acordo com um esquema múltiplo Var Aco. No componente Var estava em vigor o critério Lag 10, o qual estabelecia que, para ser reforçada, a sequência tinha que diferir das 10 últimas sequências emitidas. No componente Aco, não havia exigência de variação, e assim, qualquer sequência poderia produzir o reforço. A probabilidade dos reforços nesse componente foi acoplada àquela obtida no componente Var. Na Fase de Eliminação estava em vigor um esquema múltiplo Extinção Extinção, de forma que nenhuma sequência produzia reforços. Na Fase de Teste, as sequências continuaram em extinção e reforços foram liberados independentemente do responder, $1,5 \mathrm{~s}$ e $10 \mathrm{~s}$ após o início de cada componente. A variabilidade no responder foi avaliada por meio do valor $U$, uma medida que indica o nível de equiprobabilidade na emissão das sequências. $\mathrm{O}$ valor $\mathrm{U}$ pode variar de 0 a 1 , sendo 0 quando apenas uma sequência é emitida e sendo 1 quando todas as sequências são emitidas com igual probabilidade. Houve restabelecimento do valor U na Fase de Teste, mas apenas no componente Var.

Além do restabelecimento, Galizio et al. (2018, Experimento 3) investigaram a ressurgência de variabilidade comportamental. Com o objetivo de separar o reaparecimento da variabilidade operante e a indução de variabilidade pela extinção, esses autores utilizaram dois grupos de pombos: um com história de variação e outro com história de repetição. Na Fase de Treino, para o grupo Var, o reforço era contingente à emissão de sequências que diferissem das oito anteriores (Lag 8); para o grupo Rep, a sequência só produzia o reforço se fosse igual a uma das três últimas sequências. Na Fase de Eliminação, os dois grupos foram expostos à contingência de repetição. Na Fase de Teste, todas as sequências estavam em extinção, para ambos os grupos. Com a suspensão dos reforços para repetição, o valor $U$ aumentou indistinguivelmente para ambos os grupos. Mas, uma vez que os pombos do grupo Rep nunca foram expostos à contingência de variação, o aumento na variabilidade para esse grupo durante a Fase de Teste foi atribuído à extinção em vigor nessa fase. $\mathrm{O}$ aumento no valor $\mathrm{U}$ do grupo Var, por outro lado, foi considerado como evidência de ressurgência da variação previamente treinada.

Os resultados dos Experimentos 2 e 3 de Galizio et al. (2018) sugerem que a variabilidade comportamental pode reaparecer após ser extinta. No entanto, algumas limitações metodológicas foram observadas nesse estudo. No Experimento 2, o valor U do componente Var diminuiu de aproximadamente 0,8 para 0,6 , enquanto o valor $\mathrm{U}$ do componente Aco manteve-se inalterado (aproximadamente 0,4) na Fase de Eliminação. Ou seja, a variabilidade não foi extinta em nenhum dos componentes, o que dificulta a identificação do aumento do valor $U$ no componente Var, observado na Fase de Teste, como um exemplo de restabelecimento (i.e., como o reaparecimento de uma 
resposta previamente extinta). No Experimento 3, o aumento no valor $U$ foi similar para os grupos Var e Rep na Fase de Teste, a despeito do grupo Rep não ter sido exposto à variação na Fase de Treino. Apesar de os autores terem argumentado que esse aumento da variação foi induzido pela extinção, mas apenas no grupo Rep, não é possível descartar a possibilidade de que o mesmo tenha ocorrido no grupo Var. Ou seja, uma vez que ambos os grupos apresentaram um responder repetitivo no final da Fase de Eliminação e já que ambos mostraram um aumento similar no valor $U$ durante a Fase de Teste, não é possível afirmar com certeza que esse aumento representa variabilidade induzida pela extinção no grupo Rep e ressurgência da variabilidade operante no grupo Var.

Dessa forma, o presente estudo consistiu em uma replicação sistemática dos experimentos 2 e 3 de Galizio et al. (2018). Foi utilizado um esquema múltiplo Var Aco. A contingência Aco foi escolhida, em detrimento da contingência Rep, em função de dois aspectos: (a) na contingência Aco, o reforçamento das sequências é intermitente, o que implica períodos de extinção e, consequentemente, a possibilidade de indução de variação (Page \& Neuringer, 1985); (b) a contingência Aco e a contingência Var (Lag 10) foram programadas em um esquema múltiplo, e quando isso ocorre, a variabilidade no componente Aco tende a ser maior do que quando esse componente é apresentado isoladamente (Hunziker et al., 1996). Assim, os animais teriam uma história de emissão de sequências variadas em ambos os componentes, embora em um deles a variação fosse operante (Lag 10) e, no outro, induzida pela extinção e/ou ocasionada pela interação entre componentes (Aco). Outra mudança efetuada diz respeito à Fase de Eliminação. Em vez de utilizar uma contingência de extinção, como Galizio et al. (2018) fizeram no Experimento 2, o presente estudo utilizou uma contingência de repetição, assim como ocorreu no Experimento 3 desses autores. $\mathrm{O}$ objetivo dessa mudança consistiu em promover a extinção de variação em ambos os componentes durante a Fase de Eliminação, o que permitiria uma análise mais clara do reaparecimento da variação na Fase de Teste. Com essas mudanças, caso houvesse um maior aumento da variação no componente Lag 10 do que no componente Aco durante a Fase de Teste, poder-se-ia afirmar, com maior confiabilidade do que no estudo de Galizio et al., que esse aumento exemplificaria o reaparecimento da variação operante, em vez de variação induzida pela extinção.

Uma consideração adicional refere-se à variabilidade entre sujeitos observada no estudo de Galizio et al. (2018). Com o objetivo de reduzir essa variabilidade indesejada e aumentar a magnitude da recaída (Liggett et al., 2018), no presente estudo foi usada a combinação dos modelos experimentais de renovação, restabelecimento e ressurgência. Sendo assim, a Fase de Teste foi caracterizada pela mudança de contexto, liberação de reforços independentes e retirada dos reforços para o comportamento alternativo, respectivamente. Além do mais, o presente estudo investigou a recaída de variabilidade com ratos, em vez de pombos, visando avaliar a generalização dos resultados obtidos por Galizio et al. (2018) para outras espécies.

\section{MÉTODO}

\section{Sujeitos}

Foram utilizadas quatro ratas Wistar, experimentalmente ingênuas (G1, G2, G3 e G4), mantidas individualmente em caixas de polipropileno, com livre acesso à água, em um biotério com ciclo luz-escuro de $12 \mathrm{~h}$ (luzes acendiam às 7:00 h). Após $1 \mathrm{~h}$ do término da sessão, os sujeitos eram alimentados com uma quantidade de ração suficiente para manter seu peso em $80 \%(+-5 \mathrm{~g})$ de seu peso livre. As sessões foram realizadas sempre no turno matutino, sete dias por semana, com duração de aproximadamente 45 minutos por sessão. O projeto foi aprovado pela Comissão de Ética no Uso Animal da Universidade de Brasília, sob o protocolo $n^{\circ} 62 / 2017$.

\section{Equipamento}

Foram utilizadas quatro câmaras de condicionamento operante Med Associates ${ }^{\circledR}$. Cada câmara estava inserida em uma caixa de isolamento acústico e visual. $\mathrm{O}$ teto e as paredes laterais das câmaras eram compostos de material acrílico transparente e os painéis de trabalho e do fundo, de inox. O painel de trabalho continha duas barras (direita e esquerda), operadas por uma força mínima de $0,25 \mathrm{~N}$, uma lâmpada acima de cada barra e um bebedouro localizado em uma abertura entre as barras direita e esquerda. Quando o bebedouro era acionado, uma concha com uma mistura de água e leite condensado $(50 \% \mathrm{vol} / \mathrm{vol})$ era introduzida na abertura por um período de $5 \mathrm{~s}$. O painel do fundo continha uma lâmpada para iluminação da caixa. A programação das condições experimentais e o registro dos dados foram realizados por um computador Pentium conectado à cada câmara de condicionamento por meio de um sistema interface Med Associates ${ }^{\circledR}$.

\section{Procedimento}

O procedimento foi composto por três fases: Treino, Eliminação e Teste. Dois tipos de contextos foram utilizados ao longo das fases, em ordem contrabalanceada entre sujeitos: no contexto $\mathrm{A}$, o teto e as paredes laterais da caixa operante eram cobertas com um papelão com listras horizontais pretas e brancas e o piso continha uma placa de acrílico transparente sobre uma borracha preta; no contexto 
B, a caixa operante padrão não foi alterada. Em todas as fases, as luzes das barras e da caixa eram apagadas durante o período de blackout (BO) e a apresentação do reforço, ambos com duração de $5 \mathrm{~s}$.

Fase de Treino. Para dois sujeitos (G1 e G2), essa fase ocorreu no contexto A, e para os demais (G3 e G4), no contexto B. Nessa fase, a tarefa consistiu em emitir sequências com seis respostas, distribuídas nas barras esquerda e direita, de acordo com o esquema múltiplo Lag 10 Aco.

A sessão era iniciada com um BO de $30 \mathrm{~s}$. Após esse período, o computador sorteava o primeiro componente da sessão e, em seguida, os componentes se alternavam. No componente Lag 10, sinalizado pela luz da caixa piscando e luzes das barras esquerda e direita apagadas, a sequência era reforçada somente quando apresentava uma ou duas mudanças entre barras (critério de mudança) ${ }^{1}$ e caso diferisse das 10 últimas sequências emitidas (critério de variação); sequências que não atendiam um ou ambos os critérios eram seguidas por um BO de $5 \mathrm{~s}$. No componente Aco, sinalizado pela luz da caixa apagada e luzes das barras acesas, não havia contingência de variabilidade em vigor, de modo que sequências com uma ou duas mudanças eram reforçadas de acordo com a probabilidade de reforços programada para esse componente (maiores detalhes sobre a programação dos reforços serão fornecidos a seguir). Havia um intervalo entre componentes (ICI) de $30 \mathrm{~s}$, durante o qual todas as luzes eram apagadas. Cada componente era apresentado quatro vezes, perfazendo um total de oito componentes por sessão, e ficava em vigor até a obtenção de cinco reforços. A sessão era encerrada após a obtenção de um total de 40 reforços ou após $45 \mathrm{~min}$, o que ocorresse primeiro.

Nas primeiras 10 sessões da Fase de Treino, a probabilidade programada (PP) de reforços nos dois componentes era igual e correspondia a 1,0 , isto é, $100 \%$ das sequências que atendiam aos critérios de mudança e/ou de variabilidade eram reforçadas. Em seguida, foi iniciado o acoplamento de reforços entre componentes, conforme o seguinte critério: a probabilidade obtida (PO) de reforços durante a sessão era calculada para ambos os componentes; se a diferença na PO entre componentes fosse maior do que $20 \%$, havia uma diminuição de 0,05 no valor da PP, na próxima sessão, para o componente que havia apresentado PO mais elevada. No decorrer dessa fase, a diminuição no valor da PP sempre ocorreu no componente Aco uma vez que este componente

1 Contingências de variação tendem a gerar não somente aumentos na emissão de sequências variadas, mas também aumentos na frequência de sequências com um número maior de mudanças entre operanda, quando comparadas com contingências sem exigência de variação, de maneira que há dúvidas sobre qual dessas variáveis é selecionada pelo reforço (Machado, 1997). No estudo de Galizio et al. (2018), uma vez que nenhuma tentativa foi feita para separar essas duas variáveis, não é possível afirmar, inequivocamente, que houve recaída da variação, em vez de recaída do número de mudanças. Para evitar essa questão, o número de mudanças foi mantido constante no presente estudo. sempre gerou um número maior de reforços se comparado ao componente Lag 10. A PP de reforços para o componente Aco nunca foi menor do que 0,5 .

O critério de estabilidade utilizado para o término dessa fase foi aplicado à porcentagem de sequências reforçadas e ao tempo de recorrência (ver detalhes dessa medida na seção de Resultados). Assim, a Fase de Treino era finalizada quando, para ambas as medidas, a diferença entre a média das três penúltimas e a média das três últimas sessões era igual a, ou menor que, $20 \%$, ou após 35 sessões, o que ocorresse primeiro. Além disso, para a medida de porcentagem de sequências reforçadas, a diferença entre as médias das últimas seis sessões de ambos os componentes deveria ser menor do que $20 \%$. Finalmente, foi realizada a inspeção visual dos dados, de modo que o término da fase só ocorria se, além dos critérios acima, não houvesse tendências crescentes e decrescentes nessas medidas durante as seis últimas sessões.

Fase de Eliminação. Nessa fase estava em vigor um esquema múltiplo Rep 3 Rep 3. Em ambos os componentes, uma sequência de seis respostas, também distribuídas nas barras esquerda e direita, era seguida pelo reforço apenas quando era igual a uma das três últimas sequências. Essa contingência, portanto, extinguia variação (o padrão alvo de respostas reforçado na Fase de Treino) e promovia repetição (um padrão alternativo de respostas). O critério de número de mudanças, descrito na Fase de Treino, permaneceu em vigor durante a Fase de Eliminação. A sinalização dos componentes foi idêntica àquela utilizada na Fase de Treino.

Houve mudança de contexto nessa fase, de modo que os sujeitos $\mathrm{G} 1$ e $\mathrm{G} 2$ foram expostos ao contexto $\mathrm{B}$, e os demais (G3 e G4), ao contexto A. O critério utilizado para finalizar a fase foi baseado no tempo de recorrência, o qual deveria ser igual a, ou menor que 2 em ambos os componentes, nas duas últimas sessões dessa fase. Os demais detalhes do procedimento foram idênticos àqueles da Fase de Treino.

Fase de Teste. O procedimento de extinção estava em vigor nessa fase, em ambos os componentes, de modo que a emissão de sequências nunca produzia reforços. Assim como nas fases de Treino e de Eliminação, a luz da caixa piscava e as luzes das barras permaneciam apagadas durante um dos componentes ("Lag 10"), e a luz da caixa permanecia apagada $e$ as luzes das barras direita e esquerda permaneciam acesas durante um dos componentes ("Aco"). Em termos técnicos, o esquema em vigor era um múltiplo Extinção Extinção; no entanto, uma vez que o objetivo do estudo era verificar se haveria recaída diferencial do responder gerado pelos componentes Lag 10 e Aco, esse esquema foi denominado de múltiplo "Lag 10" "Aco", respectivamente, com base na sinalização desses componentes na Fase de Treino.

Foram liberados dois reforços, independentemente do responder, após $2 \mathrm{~s}$ e após $30 \mathrm{~s}$ desde o início da primeira apresentação de cada componente, totalizando quatro reforços por sessão. Para que os reforços independentes fossem liberados, nenhuma resposta deveria ocorrer no período de 1 
s antes da liberação programada; caso uma resposta ocorresse nesse período, o reforço era atrasado por $2 \mathrm{~s}$.

A duração de cada componente foi calculada tendo como base a última sessão da Fase de Treino. Isto é, inicialmente foi calculada a duração média do componente Lag 10 e a duração média do componente Aco nessa sessão; em seguida, foi calculada a média das durações médias dos dois componentes; finalmente, o valor obtido foi utilizado para programar a duração de cada componente na Fase de Teste. Todos os sujeitos retornaram ao contexto utilizado na Fase de Treino, caracterizando um procedimento de renovação ABA para os sujeitos G1 e G2, e renovação BAB para os sujeitos G3 e G4. Os animais foram mantidos nessa fase até que o número de sequências emitidas fosse menor do que 10 sequências, em ambos os componentes. Os demais detalhes do procedimento foram iguais àqueles da Fase de Treino.

\section{RESULTADOS}

A parte superior da Tabela 1 apresenta a porcentagem de sequências reforçadas, obtida dividindo-se o número de sequências reforçadas pelo total de sequências emitidas no componente, sendo o quociente multiplicado por 100. Nas fases de Treino e Eliminação, a porcentagem de sequências reforçadas foi semelhante entre os dois componentes, para todos os sujeitos (com exceção do sujeito G1, na Fase de Eliminação, para o qual essa porcentagem foi maior no componente previamente correlacionado com Aco), o que indica a efetividade do acoplamento de reforços. Os valores da porcentagem de reforços variaram em torno de $40 \%$ na Fase de Treino, e em torno de $66 \%$ (sujeitos G1 e G2) e $44 \%$ (sujeitos G3 e G4) na Fase de Eliminação. Não há dados para a Fase de Teste já que a emissão de sequências estava em extinção.

A parte central da Tabela 1 mostra a taxa de respostas, obtida dividindo-se o número de respostas nas barras esquerda e direita pela duração do componente. Na Fase de Treino, a taxa de respostas foi similar entre componentes (entre $23 \mathrm{e}$
52 respostas por minuto). Na Fase de Eliminação, as taxas de respostas mantiveram-se similares entre componentes (com exceção do sujeito G1, que apresentou taxas maiores no componente Rep previamente correlacionado com Lag 10 ), e próximas àquelas observadas na fase anterior. $\mathrm{Na}$ Fase de Teste, as taxas de respostas diminuíram em ambos os componentes, com exceção do sujeito G1, que apresentou aumento da taxa de respostas no componente "Aco". Nessa fase, todos os sujeitos apresentaram taxas de respostas maiores no componente "Lag 10", com exceção do sujeito G3, para o qual não houve diferença entre componentes.

A parte inferior da Tabela 1 apresenta a porcentagem de sequências com 1-2 mudanças em cada componente do esquema múltiplo. Essa medida foi obtida dividindo-se o número de sequências com 1-2 mudanças pelo total de sequências emitidas no componente, sendo o quociente multiplicado por 100. Na Fase de Treino, para todos os sujeitos, a porcentagem de sequências com 1-2 mudanças

Tabela 1

Porcentagem de Sequências Reforçadas, Taxa de Respostas e Porcentagem de Sequências com 1-2 Mudanças na Última Sessão das Fases de Treino e Eliminação e na Primeira Sessão da Fase de Teste, em Cada Componente do Esquema múltiplo, para Cada Sujeito.

\begin{tabular}{|c|c|c|c|c|c|c|}
\hline & \multicolumn{2}{|c|}{ Treino } & \multicolumn{2}{|c|}{ Eliminação } & \multicolumn{2}{|c|}{ Teste } \\
\hline & Lag 10 & Aco & Rep ("Lag 10") & Rep (“Aco") & "Lag 10" & "Aco" \\
\hline \multicolumn{7}{|c|}{ Sequências Reforçadas (\%) } \\
\hline G1 & 39 & 38 & 54 & 69 & -- & -- \\
\hline G2 & 44 & 36 & 69 & 74 & -- & -- \\
\hline G3 & 36 & 43 & 41 & 47 & -- & -- \\
\hline G4 & 44 & 38 & 47 & 41 & -- & -- \\
\hline \multicolumn{7}{|c|}{ Taxa de Respostas } \\
\hline G1 & 52 & 46 & 52 & 33 & 17 & 42 \\
\hline G2 & 33 & 31 & 27 & 26 & 6 & 20 \\
\hline G3 & 27 & 23 & 33 & 25 & 23 & 25 \\
\hline G4 & 42 & 38 & 31 & 32 & 12 & 22 \\
\hline \multicolumn{7}{|c|}{ Sequências com 1-2 Mudanças (\%) } \\
\hline G1 & 90 & 86 & 86 & 67 & 81 & 77 \\
\hline G2 & 91 & 82 & 87 & 76 & 91 & 71 \\
\hline G3 & 81 & 61 & 58 & 51 & 55 & 69 \\
\hline G4 & 83 & 84 & 65 & 60 & 77 & 86 \\
\hline
\end{tabular}

Nota. "Lag 10" e "Aco" = componentes sinalizados pelos estímulos exteroceptivos correlacionados com os componentes Lag e Aco, respectivamente, durante a Fase de Treino. Os dados de porcentagem de sequências reforçadas na Fase de Teste não foram apresentados uma vez que extinção estava em vigor em ambos os componentes. 
foi similar entre componentes (entre 81 e 90\%), com exceção do sujeito G3, para o qual essa porcentagem foi igual a $61 \%$ no componente Aco. Na Fase de Eliminação, embora as porcentagens de sequências com 1-2 mudanças tenham diminuido para os sujeitos G3 e G4, assumindo valores entre 51 e $65 \%$, permaneceram comparáveis entre componentes (com exceção do sujeito G1, o qual apresentou uma porcentagem maior no componente Rep previamente correlacionado com Lag 10). Na Fase de Teste, as alterações nas porcentagens de sequências com 1-2 mudanças foram assistemáticas entre sujeitos e entre componentes, apresentando valores entre 55 e $91 \%$.

A Figura 1 apresenta a porcentagem de sequências corretas (i.e., acurácia) em cada componente do esquema múltiplo, nas últimas seis sessões da Fase de Treino e em todas as sessões das fases de Eliminação e Teste, para cada sujeito. Essa medida foi obtida dividindo-se o número de sequências corretas pelo total de sequências emitidas no componente, sendo o quociente multiplicado por 100 . Durante as fases de Treino e de Eliminação, sequências corretas são aquelas que atenderam o critério de mudança em ambos os componentes. Adicionalmente, também cumpriram o critério de variação no componente Lag 10 (Fase de Treino) e o critério de repetição em ambos componentes (Fase de Eliminação). Na Fase de Teste, sequências corretas são aquelas que atenderiam às contingências da Fase de Treino, caso essas contingências estivessem operando na última fase. No componente Aco (Fase de Treino), sequências corretas não correspondem às sequências reforçadas devido ao acoplamento dos reforços entre componentes. Isto porque, nesse componente, embora todas as sequências com 1-2 mudanças fossem corretas, o reforçamento dessas sequências ocorria de acordo com a probabilidade do reforço no componente Lag 10 da sessão imediatamente anterior.

$\mathrm{Na}$ Fase de Treino, a porcentagem de sequências corretas foi maior no componente Aco do que no componente Lag 10, para todos os sujeitos. Com a mudança, na Fase de Eliminação, para a contingência de repetição, houve uma diminuição inicial nas porcentagens de sequências corretas, principalmente no componente sinalizado com os mesmos estímulos exteroceptivos usados no componente Aco da fase anterior, de modo que essas porcentagens se tornaram similares entre componentes já na primeira (sujeitos G2, G3 e G4) ou segunda (sujeito G1) sessões; com a exposição à contingência de repetição, a porcentagem de sequências corretas aumentou, atingindo valores intermediários àqueles observados nos dois componentes da fase anterior. $\mathrm{Na}$ Fase de Teste, a porcentagem de sequências corretas no componente "Aco" foi maior do que no componente "Lag 10 ", com exceção do sujeito G2 que apresentou porcentagens similares entre componentes.

A Figura 2 apresenta uma medida de variabilidade, denominada por Barba (2012) de tempo de recorrência. Essa medida mostra o número de sequências emitidas entre a ocorrência de uma determinada sequência e sua próxima ocorrência, ou seja, indica recência. Por exemplo, se o

\section{ACURÁCIA}

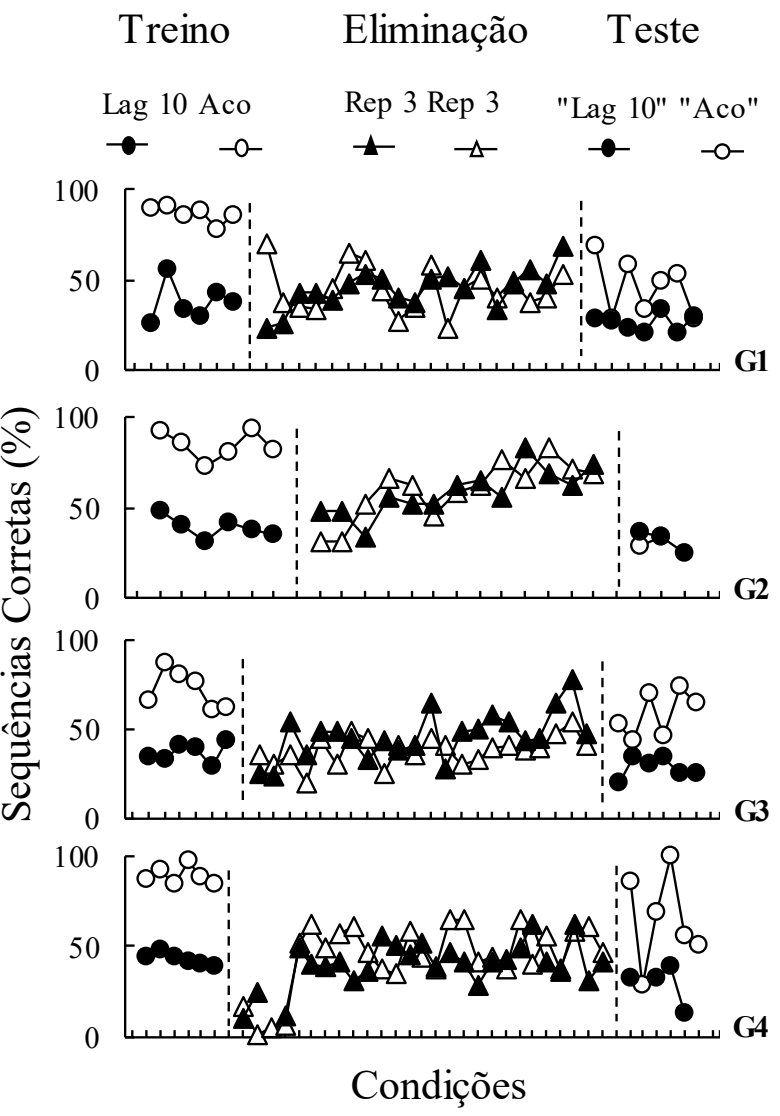

Figura 1. Porcentagem de sequências corretas em cada componente do esquema múltiplo, nas últimas seis sessões da Fase de Treino e em todas as sessões das fases de Eliminação e Teste, para cada sujeito. Na Fase de Teste, sequências corretas são aquelas que atenderiam às contingências da Fase de Treino caso estivessem em vigor nessa última fase.

organismo emitir as sequências EDDDD, DDDDE, DDDDD e EDDDD, o tempo de recorrência para a última sequência (EDDDD) seria igual a dois, uma vez que duas sequências foram emitidas após a primeira emissão da sequência EDDDD e sua próxima emissão; o tempo de recorrência para as sequências DDDDE e DDDDD seria zero, pois essas sequências foram emitidas somente uma vez (a primeira sequência emitida não tem tempo de recorrência). Assim, quanto maior o tempo de recorrência, maior a variação na emissão das sequências². Sob o critério Lag 10, por exemplo,

2 Apesar de o valor U ser a variável dependente mais comum em pesquisas de variabilidade operante, essa medida não foi utilizada no presente estudo por dois motivos. Primeiro, o valor U é afetado quando um número reduzido de sequências é emitido (e.g., Galizio et al., 2018; Kong et al., 2017). Assim, como a Fase de Teste envolveu extinção e, consequentemente, poucas sequências ocorreram nessa fase, o valor U não é uma medida recomendável. Segundo, o critério lag seleciona a recência na emissão das sequências, enquanto o valor $U$ expressa a equiprobabilidade na emissão dessas sequências. Dessa forma, o tempo de recorrência, o qual informa sobre recência entre a ocorrência passada e atual de uma sequência, seria uma medida mais adequada quando critérios lag são utilizados (Barba, 2012). 
o reforço seleciona diretamente o tempo de recorrência igual a 10 , uma vez que uma sequência só é reforçada se diferir das 10 sequências imediatamente anteriores.

No presente estudo, o tempo de recorrência médio foi calculado para os dois componentes do esquema múltiplo, para cada sujeito. Para tanto, todos os tempos de recorrência de uma sessão foram divididos pelo número de sequências emitidas nessa mesma sessão, excluindo-se a primeira sequência. Na Fase de Treino, o tempo de recorrência não diferiu entre componentes na maioria das sessões de todos os sujeitos. Na Fase de Eliminação, o tempo de recorrência diminuiu similarmente nos dois componentes ao longo da exposição à contingência de repetição, atingindo valores próximos a 0,0 . Na Fase de Teste, com a retirada da contingência de repetição, houve um aumento no tempo de recorrência no componente "Lag 10 ", mas não no componente "Aco", durante o qual o tempo de recorrência manteve-se próximo a 0,0 .

Cançado et al. (2016) distinguiram duas análises da recaída comportamental - a análise da recuperação e a análise do reaparecimento do responder -, cada uma

Figura 2. Tempo de recorrência médio, em cada componente do esquema
múltiplo, nas seis últimas sessões da Fase de Treino e em todas as sessões

Figura 2. Tempo de recorrência médio, em cada componente do esquema
múltiplo, nas seis últimas sessões da Fase de Treino e em todas as sessões das fases de Eliminação e Teste, para cada sujeito.

\section{VARIAÇÃO}

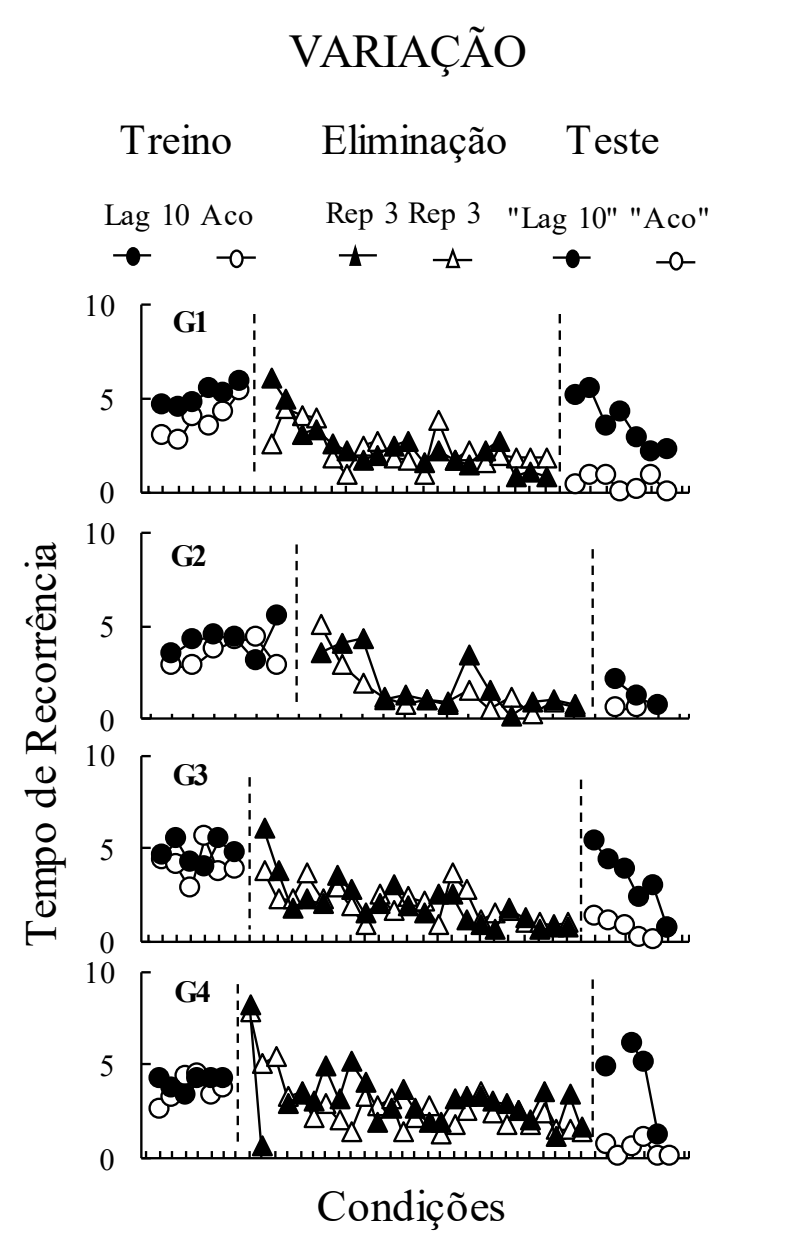

apresentando uma informação específica sobre o fenômeno. $\mathrm{Na}$ análise da recuperação do responder, os valores obtidos na Fase de Teste são divididos pelos valores obtidos na Fase de Treino (TT/TR). Essa análise indica o quanto o responder emitido durante a Fase de Teste se aproxima daquele emitido durante a Fase de Treino. Assim, quanto mais próximos de 1,0 forem as proporções, maior a similaridade entre o responder nas fases de Teste e Treino e, assim, maior a recuperação. $\mathrm{Na}$ análise do reaparecimento do responder, os valores observados na Fase de Teste são divididos por aqueles obtidos no final da Fase de Eliminação (TT/EL). Essa análise mostra o quanto o responder reapareceu na Fase de Teste após ter sido extinto na Fase de Eliminação. Proporções iguais a 1,0 indicam que o responder não diferiu entre as fases de Teste e de Eliminação (não houve reaparecimento), proporções maiores do que 1,0 indicam que o responder foi mais acentuado na Fase de Teste do que na Fase de Eliminação (houve reaparecimento) e proporções menores do que 1,0 mostram o inverso.

A Figura 3 apresenta as análises de recaída do tempo de recorrência. Para a realização dessas análises, a média

\section{RECAIIDA}

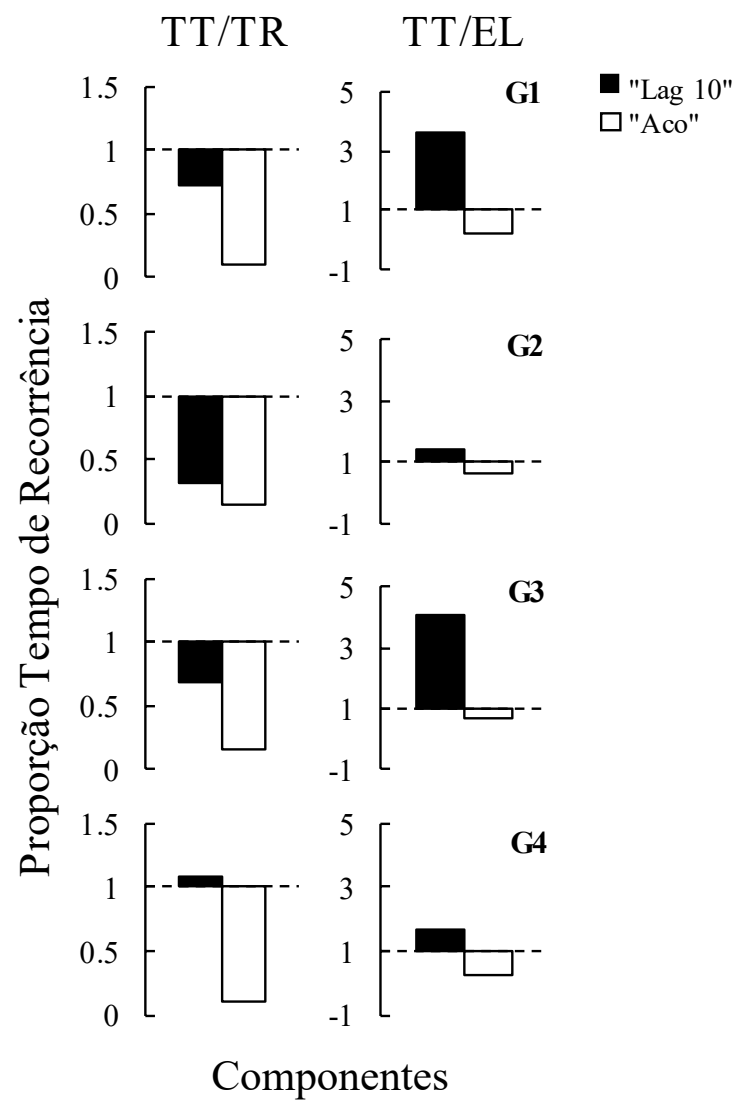

Figura 3. Tempo de recorrência médio na Fase de Teste (TT) como proporção do tempo de recorrência médio nas fases de Treino (TR) e Eliminação (EL) em cada componente do esquema múltiplo, para cada sujeito (ver texto para detalhes). 
do tempo de recorrência médio de todas as sessões da Fase de Teste foi dividida pela média do tempo de recorrência médio das seis últimas sessões da Fase de Treino (TT/TR) e das duas últimas sessões da Fase de Eliminação (TT/EL). A análise TT/TR mostra que, para todos os sujeitos, houve maior recuperação no componente "Lag 10" se comparado ao componente "Aco". A análise TT/EL mostrou resultados consistentes com aqueles da análise TT/TR, ou seja, houve reaparecimento do tempo de recorrência, mas somente no componente "Lag 10", para todos os sujeitos.

\section{DISCUSSÃO}

O objetivo desse estudo foi verificar se um padrão comportamental variável, estabelecido por reforçamento direto da variação (componente Lag 10), ou induzido pela intermitência do reforço e/ou interação entre componentes (componente Aco) e, posteriormente, extinto, reapareceria sob condições ambientais que envolvessem mudança do contexto em que a extinção foi estabelecida (Renovação), descontinuação de reforços para uma resposta alternativa (Ressurgência) e liberação de reforços independentes do responder (Restabelecimento).

Com relação à aquisição de variação (Fase de Treino) e repetição (Fase de Eliminação), os resultados mostram que: (1) a porcentagem de sequências reforçadas, taxa de respostas e porcentagem de sequências com 1-2 mudanças foram similares entre componentes na maioria das sessões; (2) a porcentagem de sequências corretas foi maior no componente que não exigia (Aco) do que no componente que exigia (Lag 10) variação, e não diferiu entre os componentes que exigiam repetição; e (3) o tempo de recorrência foi próximo entre os componentes, mas diferiu entre fases, apresentando valores maiores nos componentes Lag 10 e Aco do que nos componentes Rep. Com relação à recaída de variação (Fase de Teste), foi observado que: (1) a recuperação do tempo de recorrência foi mais acentuada no componente "Lag 10"do que no componente "Aco"; e (2) o reaparecimento do tempo de recorrência só ocorreu no componente "Lag 10".

\section{Aquisição de Variação e Repetição}

$\mathrm{Na}$ Fase de Treino, a porcentagem de sequências corretas foi maior no componente Aco se comparado ao componente Lag 10. Nos estudos que utilizam a contingência Aco, qualquer sequência, independentemente da variação no responder, gera reforços e, assim, não há erros. Dada a obviedade dos resultados (todas as sequências são consideradas corretas), essa medida não é comumente incluída na análise dos dados (e.g., Denney \& Neuringer, 1998; Galizio et al., 2018; Morgan \& Neuringer, 1990; Page \& Neuringer, 1985). No presente estudo, no entanto, essa análise é relevante uma vez que nem toda sequência produzia reforços no componente Aco. Ou seja, o reforço ocorria apenas quando a sequência apresentava 1-2 mudanças entre operanda. Assim, uma alta porcentagem de sequências corretas nesse componente, como foi aqui observado, indica o atendimento do critério de mudança. A menor acurácia no componente Lag 10 sugere que a inclusão do critério de variação "dificultou" a obtenção de reforços. Essa sugestão parece plausível quando se considera que, embora a porcentagem de sequências com 1-2 mudanças tenha sido similar entre componentes, o tempo de recorrência no componente Lag 10 (i.e., TRs entre 3 e 5) foi menor do que aquele diretamente reforçado pela contingência de variabilidade (i.e., 10). Ou seja, os erros cometidos no componente Lag 10 foram ocasionados, em sua maioria, pela exigência de variação, e não pela exigência de 1 ou 2 mudanças entre operanda.

O tempo de recorrência não diferiu entre componentes, a despeito de haver ou não exigência de variação para a liberação de reforços. A similaridade nos níveis de variabilidade entre componentes pode ter ocorrido devido à interação entre as contingências em vigor no esquema múltiplo (Reynolds, 1961). No componente Lag 10, pelo menos 11 sequências diferentes com 1-2 mudanças eram necessárias para a produção de reforços; no componente Aco, o reforço poderia ser produzido por meio da emissão de uma única sequência com 1-2 mudanças. A influência do componente Aco sobre o componente Lag 10 era bastante improvável, uma vez que a emissão de apenas uma sequência ou de poucas sequências diferentes não produziria reforços no componente Lag 10. Por outro lado, a variação na emissão de sequências, determinada pela contingência Lag 10, era também efetiva na produção de reforços no componente Aco. Neste último componente, em caso de controle máximo da contingência em vigor, o tempo de recorrência deveria apresentar valores iguais (ou próximos) a 0,0 . No entanto, os valores obtidos foram superiores a 0,0 e próximos àqueles observados no componente Lag 10. Isso indica que a variabilidade no componente Aco foi bem maior do que a esperada e sugere que a contingência de variação no componente Lag 10 afetou o desempenho no componente Aco. Interação entre componentes também foi observada por Aló e Abreu-Rodrigues (2017) ao exporem ratos a esquemas múltiplos: a variabilidade no componente Aco foi sistematicamente maior quando esse componente foi acompanhado pelo componente Var do que quando foi acompanhado pelo componente Rep.

Na Fase de Eliminação, a contingência de repetição em vigor gerou porcentagens de sequências corretas maiores e tempo de recorrência menores do que aqueles obtidos no componente Lag 10 da fase anterior, um resultado compatível com aqueles relatados por Page \& Neuringer (1985) ao manipularem os valores do critério lag em 
fases experimentais distintas. Maior acurácia e menor variabilidade no componente Rep do que no componente Var também foram encontradas quando essas contingências foram programadas nos componentes de um esquema múltiplo, em estudos com pombos (e.g., Galizio et al., 2018; Odum et al., 2006), ou nos elos terminais de um esquema concorrente, tanto em estudos com pombos (AbreuRodrigues et al., 2005) quanto com humanos jovens e idosos (Natalino-Rangel, 2010). Em conjunto, esses resultados indicam que a variabilidade pode ser diretamente produzida por contingências de reforço.

\section{Recaída da Variabilidade}

Duas variáveis de controle têm sido amplamente investigadas em estudos de recaída: taxa de respostas e taxa de reforços. Esses estudos apontam, consistentemente, que quanto maior a taxa da resposta alvo durante a Fase de Treino, maior a magnitude da recaída. (e.g., Berry et al., 2014; da Silva et al., 2008; Doughty et al., 2004; MirandaDukosky et al., 2015). Com relação à taxa de reforços da resposta alvo, alguns estudos também apontam uma relação direta (e.g., Berry et al., 2014; Miranda-Dukoski et al., 2015), enquanto outros apontam uma relação assistemática (e.g., da Silva et al., 2008) entre essa variável e a recaída. No presente estudo, foi observada recaída diferencial do responder aprendido sob contingências que exigiam (Lag 10) ou não exigiam (Aco) variação, como será discutido a seguir, mas essa diferença não pode ser atribuída à taxa de reforços ou à taxa de respostas uma vez que essas medidas foram similares entre componentes na Fase de Treino.

Em termos do tempo de recorrência, as análises TT/ TR e TT/EL mostraram resultados consistentes, ou seja, tanto a recuperação quanto o reaparecimento do tempo de recorrência foram mais acentuados no componente "Lag 10" para todos os sujeitos. Essa recaída diferencial entre componentes pode ser atribuída à história de reforçamento diferencial do tempo de recorrência durante a Fase de Treino. No presente estudo, o critério Lag 10 estabelecia que sequências com recência igual ou maior que 10 produziriam o reforço; caso contrário, haveria um $\mathrm{BO}$. No componente Aco, por outro lado, não havia reforçamento diferencial do tempo de recorrência já que a única exigência para a liberação do reforço era a emissão de uma sequência com 1-2 mudanças. Diante disso, seria esperado que a recaída do tempo de recorrência, caso ocorresse, fosse mais acentuada no componente "Lag 10", em comparação com o componente "Aco", uma vez que o tempo de recorrência foi diretamente reforçado no componente Lag 10 durante a Fase de Treino.

Um dos problemas encontrados em estudos de variabilidade que utilizam extinção é a possibilidade de que os níveis de variabilidade observados não sejam produzidos pelo reforçamento direto da variação, mas sim, induzidos pela extinção. Dessa forma, no presente estudo, pode ser levantada a seguinte questão: o aumento maior do tempo de recorrência no componente "Lag 10" deve ser considerado como recaída da variabilidade anteriormente reforçada, conforme acima proposto, ou como variabilidade induzida pela retirada dos reforços para o responder repetitivo? Não é possível refutar a possibilidade de que parte da variabilidade observada na Fase de Teste tenha sido induzida pela extinção. No entanto, considerando que (a) não há justificativa óbvia para que essa indução tenha sido mais acentuada no componente "Lag 10" do que no componente "Aco"; (b) o tempo de recorrência foi diretamente reforçado no componente Lag 10, mas não no componente Aco; (c) a variabilidade foi extinta nos dois componentes na Fase de Eliminação; e (d) o aumento no tempo de recorrência foi maior no componente com história de reforçamento, parece mais plausível considerar esse aumento como um exemplo de recaída de variabilidade operante. Essa afirmativa é apoiada pela literatura (e.g., Epstein, 1983; da Silva et al., 2008), a qual ressalta o papel da história de reforçamento no fenômeno da recaída. Esses estudos mostram que quando um comportamento reforçado recentemente é exposto à extinção, vários outros comportamentos podem ser induzidos pela extinção, mas aqueles com história prévia de reforçamento são os mais prováveis de ocorrer.

A robustez dessa interpretação é maior no presente estudo, quando comparado ao de Galizio et al. (2018). Isto porque, no estudo desses autores, a variabilidade não foi extinta em ambos os componentes na Fase de Eliminação, as interpretações dos resultados foram feitas com base em análises estatísticas de diferenças entre médias e houve muita inconsistência entre sujeitos. No presente estudo, por outro lado, houve uma redução acentuada na variabilidade durante a Fase de Eliminação, os dados foram analisados individualmente e os resultados foram consistentes entre sujeitos. É possível que a redução na inconsistência entre sujeitos, aqui observada, tenha sido ocasionada pela combinação de três variáveis de controle da recaída na Fase de Teste: mudança de contexto, liberação de reforços independentes do responder e extinção da extinção.

Os resultados do presente estudo são consistentes com a literatura que mostra que operantes compostos por uma sequência de respostas são suscetíveis à recaída, assim como operantes compostos por uma única resposta. A recaída de sequências de respostas já havia sido observada com sequências de duas (e.g., Bachá-Mendez et al., 2007), três (e.g., Pontes \& Abreu-Rodrigues, 2016, Experimento 2; Sanchéz-Carrasco \& Nieto, 2005), quatro (Galizio et al., 2018) e cinco (Pontes \& Abreu-Rodrigues, 2016, Experimento 1) respostas. O presente estudo estende esses resultados ao demonstrar que sequências compostas por seis respostas também podem reaparecer.

Galizio et al. (2018) mostraram que o fenômeno da recaída pode ser verificado não somente por meio de medidas comportamentais como taxa e latência da resposta, mas também por meio de medidas de variabilidade, mais especificamente, o valor U. Uma contribuição adicional 
do presente estudo, então, é a demonstração de que outras medidas de variabilidade (i.e., tempo de recorrência) também são adequadas para a investigação do fenômeno de recaída. Finalmente, o presente estudo mostra que os resultados de Galizio et al., obtidos com pombos, são generalizáveis para ratos.
Investigações futuras poderiam incluir valores diferenciados do critério lag nos componentes do esquema múltiplo em vigor na Fase de Treino (e.g., Lag 2 e Lag 10), o que permitiria avaliar se a magnitude da recaída seria diferencialmente afetada pelos níveis de variabilidade previamente treinados.

\section{REFERÊNCIAS}

Abreu-Rodrigues, J., Lattal, K. A., dos Santos, C. V., \& Matos, R. A. (2005). Variation, repetition, and choice. Journal of the Experimental Analysis of Behavior, 83, 147-168.

Aló, R. M., \& Abreu-Rodrigues (2017, Outubro). The resistance to change of different levels of behavioral variability. In J. Abreu-Rodrigues (Chair), Behavioral history in contexts of self-control, relapse, and resistance to change. Symposium conducted at the meeting of the 9th International Conference of the Association for Behavior Analysis, Paris - França.

Bachá-Méndez, G., Reid, A. K., \& Mendoza-Soylovna, A. (2007). Resurgence of integrated behavioral units. Journal of the Experimental Analysis of Behavior, 87, 5-24.

Barba, L. S. (2012). Operant variability: A conceptual analysis. The Behavior Analyst, 35, 213-227.

Berry, M. S., Sweeney, M. M., \& Odum, A. L. (2014). Effects of baseline reinforcement rate on operant $\mathrm{ABA}$ and $\mathrm{ABC}$ renewal. Behavioural Processes, 108, 87-93.

Bouton, M. E., \& Trask, S. (2016). Role of the discriminative properties of the reinforcer in resurgence. Learning \& Behavior, 44, 137-150.

Cançado, C. R. X., Abreu-Rodrigues, J., \& Aló, R. M. (2016). A note on measuring recurrence. Revista Mexicana de Análisis de la Conducta, 42, 75-86.

Craig, A. R., Browning, K. O., Nall, R. W., Marshall, C. M., \& Shahan, T. A. (2017). Resurgence and alternative-reinforcer magnitude. Journal of the Experimental Analysis of Behavior, 107, 218-233.

da Silva, S. P. D., Maxwell, M. E., \& Lattal, K. A. (2008). Concurrent resurgence and behavioral history. Journal of the Experimental Analysis of Behavior, 90, 313-331.

Denney, J., \& Neuringer, A. (1998). Behavioral variability is controlled by discriminative stimuli. Animal Learning \& Behavior, 26, 154-162.

Doughty, A. H., Reed, P., \& Lattal, K. A. (2004). Differential reinstatement predicted by pre-extinction response rate. Psychonomic Bulletin and Review, 11, 1118-1123.

Epstein, R. (1983). Resurgence of previously reinforced behavior during extinction. Behaviour Analysis Letters, 3, 391-397.

Galizio, A., Frye, C. C., Haynes, J. M., Friedel, J. E., Smith, B. M., \& Odum, A. L. (2018). Persistence and relapse of reinforced behavioral variability. Journal of the Experimental Analysis of Behavior, 109, 210-237.

Hunziker, M. H. L., Saldana, R. L., \& Neuringer, A. (1996). Behavioral variability in SHR and WKY rats as a function of rearing environment and reinforcement contingency. Journal of the Experimental Analysis of behavior, 65, 129-144.

Kong, X., McEwan, J. S., Bizo, L. A., \& Foster, T. M. (2017). An analysis of U-value as a measure of variability. The Psychological Record, 67, 581-586.

Liggett, A. P., Nastri, R., \& Podlesnik, C. A. (2018). Assessing the combined effects of resurgence and reinstatement in children diagnosed with autism spectrum disorder. Journal of the Experimental Analysis of Behavior, 109, 408-421.

Machado, A. (1997). Increasing the variability of response sequences in pigeons by adjusting the frequency of switching between two keys. Journal of the Experimental Analysis of Behavior, 68, 1-25.

Miranda-Dukoski, L., Bensemann, J., \& Podlesnik, C. A. (2015). Training reinforcement rates, resistance to extinction, and the role of context in reinstatement. Learning \& Behavior, 44, 29-48.

Morgan, L. \& Neuringer, A. (1990). Behavioral variability as a function of response topography and reinforcement contingency. Animal Learning \& Behavior, 18, 257-263.

Natalino-Rangel, P. C. (2010). Variabilidade comportamental: uma comparação entre pessoas idosas e jovens (Tese de Doutorado). Universidade de Brasília, Brasília.

Nighbor, T. D., Oliver, A. C., \& Lattal, K. A. (2020). Resurgence without overall worsening of alternative reinforcement. Behavioural Processes, 179, 104219.

Odum, A. L., Ward, R. D., Barnes, C. A., \& Burke, K. A. (2006). The effects of delayed reinforcement on variability and repetition of response sequences. Journal of the Experimental Analysis of Behavior, 86, 159-179.

Page, S., \& Neuringer, A. (1985). Variability is an operant. Journal of Experimental Psychology: Animal Behavior Processes, 11, 429-452.

Podlesnik, C. A., Kelley, M. E., Jimenez-Gomez, C., \& Bouton, M. E. (2017). Renewed behavior produced by context change and its implications for treatment maintenance: A review. Journal of Applied Behavior Analysis, 50, 675-697.

Pontes, T. N. R., \& Abreu-Rodrigues, J. (2016). Reappearance of sequences with different number of responses in extinction and variation contexts. Psicologia: Teoria e Pesquisa, 32(esp), $1-12$.

Reid, R. L. (1958). The role of the reinforcer as a stimulus. British Journal of Psychology, 49, 202-209.

Reynolds, G. S. (1961). Behavioral contrast. Journal of the Experimental Analysis of Behavior, 4, 57-71.

Sánchez-Carrasco, L. S., \& Nieto, J. (2005). Resurgence of three response sequences in rats. Revista Mexicana de Análisis de la Conducta, 31, 215-226.

Souza, A., \& Abreu-Rodrigues, J. (2010). Discriminative proprieties of vary and repeat contingencies. Behavioural Processes, $85(2), 116-125$.

Skinner, B. F. (1950). Are theories of learning necessary? Psychological Review, 57, 193-216.

Todd, T. P., Winterbauer, N. E., \& Bouton, M. E. (2012). Effects of the amount of acquisition and contextual generalization on the renewal of instrumental behavior after extinction. Learning \& Behavior, 40, 145-157. 